rable to that seen by Sugase and colleagues ${ }^{1}$. However, the activity described by Sugase and colleagues seems difficult to attribute to behavioral influences, because the animals were not required to make any behavioral response but merely viewed the stimuli passively.

The above examples illustrate several coding strategies: multiple response measures encoding the same stimulus, multiple response measures encoding multiple stimulus attributes, a single response measure encoding combinations of attributes, or a single response encoding a stimulus and its behavioral significance. In contrast to all of these examples, Sugase and colleagues have shown that a single response measure can evolve over time to encode new information, in the absence of any external cues.

How might this increase in information content come about? Computational models of visual processing often make use of feedback and lateral inhibition to enhance weak or noisy signals ${ }^{10,11}$. These models receive some experimental support from recordings in primary visual cortex ${ }^{12-14}$, but they can only work in cases where the information for the feedback or inhibitory signals is already present in the responses. It seems unlikely that local feedback or lateral inhibition could explain the results of Sugase and colleagues, because they found very few neurons that contained any detectable information about face identity or expression during the early phase of the response. Instead, this information must be introduced via top-down inputs from other brain areas. Top-down processes are typically used by modelers to allow sensory processing to be modulated by information not present in the initial responses, such as behavioral significance or semantic knowledge. However, they can also be used in models involving reciprocal interactions between different sensory areas, in which signals from one area are conveyed to another area where new information from another source is added. The resultant signal containing the new information, which might reflect other sensory processes, memory or behavioral significance, can then be sent back to modulate activity in the earlier area. In the present example, computations in the inferior temporal cortex would establish the presence of a face, and this information would be passed to other areas that would extract information about identity and facial expression. As the authors discuss, the inferior temporal cortex has many connections to and from cortical and subcortical areas that are implicated in social and emotional processing. The delay of some $50 \mathrm{~ms}$ between the signal that a face was present and the appearance of information about identity or expression is also consistent with the idea that the initial signal from the temporal cortex passes to other regions that process it further and then relay the resulting signals back to the temporal cortex.

As with any innovative and exciting study, the findings of Sugase and colleagues raise more questions than they answer. Where does the 'new' information about identity and expression come from? Does the total amount of information encoded by the inferior temporal neurons increase over time, or does the new information replace the old? Assuming that the new information is introduced from elsewhere in the brain, how does it affect the processing that occurs within the inferior temporal cortex itself? Ultimately, it will be important to understand the relationship between this neural activity and behavior. For instance, does the discriminatory behavior of these neurons match the ability of the monkey to make behavioral discriminations? Can the monkey's behavior be altered by disrupting this topdown processing, for instance using selective lesions or microstimulation? If it turns out that the changes in information content over time have functional significance, then our understanding of how brain areas process information, both locally and in combination with other areas, will have been substantially advanced.

1. Sugase, Y., Yamane, S., Ueno, S. \& Kawano, K. Nature 401 (in press).

2. Optican, L. \& Richmond, B. J. J. Neurophysiol. 57, 162-178 (1987).

3. Gawne, T. J., Kjaer, T. W. \& Richmond, B. J. J. Neurophysiol. 76, 1356-1360 (1996).

4. Worgotter, F., Opara, R., Funke, K. \& Eysel, U. Neuroreport 7, 741-744 (1996).

5. Tanaka, K., Saito, H.-A., Fukada, Y. \& Moriya, M. J. Neurophysiol. 66, 170-189 (1991).

6. Oram, M. W. \& Perrett, D. I. J. Neurophysiol. 76, 109-129 (1996).

7. Oram, M. W. \& Perrett, D. I. J. Neurophysiol. 68, 70-84 (1992).

8. Desimone, R. \& Duncan, J. Annu. Rev. Neurosci. 18, 193-222 (1995).

9. Chelazzi, L., Miller, E. K., Duncan, J. \& Desimone, R. Nature 363, 345-347 (1993).

10. Grossberg, S. Cognit. Sci. 11, 23-63 (1987).

11. McClelland, J. L. \& Rumelhart, D. E. Explorations in Parallel Distributed Processing (MIT Press, Cambridge, Massachusetts, 1988).

12. Sillito, A. M. J. Physiol. (Lond.) 250, 305-329 (1975).

13. Berman, N. J., Douglas, R. J., Martin, K. A. C. \& Whitteridge, D. J. Physiol. (Lond.) 440, 697-722 (1991).

14. Frith, C. \& Dolan, R. J. Phil. Trans. R. Soc. Lond. B 352, 1221-1230 (1997).

\title{
Switching the hippocampus off and on
}

Memory consists of interdependent but dissociable processes, such as encoding, storage, consolidation and retrieval. Lesion studies have shown that the hippocampus is necessary for declarative memory, but pinning down its exact contribution to these individual stages has proved tricky. Because brain lesions are permanent, they cannot unambiguously dissociate the stages of memory.

Richard Morris and his colleagues (University of Edinburgh Medical School) have now developed a technique that could overcome this problem. On page 898, they report reversible, temporary inactivation of the hippocampus with LY326325, a selective water soluble antagonist of AMPA/kainate glutamate receptors. The authors infused the dorsal hippocampus (inclusive of areas CA1-CA3 and the dentate gyrus) of rats with this drug during training and/or retention on a version of the water maze test. The authors verified physiologically that they could selectively 'switch off' the dorsal hippocampus for varying periods and then switch it on again and have it work normally afterward. This technique revealed that the hippocampus is critical for both encoding and retrieval of spatial memory. This approach represents a potentially powerful way to test whether the functional integrity of various brain areas is necessary for specific memory processes.

\section{Kalyani Narasimhan}

